\title{
On the existence of Diophantine quadruples in $Z[\sqrt{(}-2)]$ \\ Ivan Soldo
}




\title{
ON THE EXISTENCE OF DIOPHANTINE QUADRUPLES IN
} $Z[\sqrt{-2}]$

\author{
IVAN SOLDO
}

Received 11 July, 2012

\begin{abstract}
By the work of Abu Muriefah, Al-Rashed, Dujella and the author, the problem of the existence of $D(z)$-quadruples in the ring $\mathbb{Z}[\sqrt{-2}]$ has been solved, except for the cases

$$
\begin{aligned}
& z=24 a+2+(12 b+6) \sqrt{-2}, z=24 a+5+(12 b+6) \sqrt{-2}, \\
& z=48 a+44+(24 b+12) \sqrt{-2} .
\end{aligned}
$$
\end{abstract}

In this paper, we present some new formulas for $D(z)$-quadruples in these remaining cases, involving some congruence conditions modulo 11 on integers $a$ and $b$. We show the existence of $D(z)$-quadruple for significant proportion of the remaining three cases.

2000 Mathematics Subject Classification: 11D09; 11R11

Keywords: diophantine quadruples, quadratic field

\section{INTRODUCTION}

Let $z$ be an element of a commutative ring $R$. A Diophantine quadruple with the property $D(z)$, or a $D(z)$-quadruple, is a set $D$ of four non-zero elements of $R$ with the property that the product of any two distinct elements of this set increased by $z$ is a square of some element in $R$. Any set $D$ satisfying this condition is called a set with the property $D(z)$.

Greek mathematician Diophantus of Alexandria found four positive rational numbers $\left\{\frac{1}{16}, \frac{33}{16}, \frac{17}{4}, \frac{105}{16}\right\}$ with the property $D(1)$ (see [4]). The first $D(1)$-quadruple in integers, the set $\{1,3,8,120\}$, was found by Fermat (see [4,15]). Baker and Davenport proved that Fermat's set cannot be extended to a $D(1)$-quintuple in integers (see [2]). In 2004, Dujella proved that in $\mathbb{Z}$ there does not exist a $D(1)$-sextuple and there are only finitely many $D(1)$-quintuples (see [8]).

Many authors considered the problem of the existence of Diophantine quadruples in integers. Brown (see [3]) proved that if $n \in \mathbb{Z}, n \equiv 2(\bmod 4)$, then there does not exist a $D(n)$-quadruple. On the other hand, Dujella (see [5]), proved that if $n \neq \equiv 2$ $(\bmod 4)$ and if $n \notin S=\{-4,-3,-1,3,5,8,12,20\}$, then there exists at least one $D(n)$-quadruple, and he conjectured that there does not exist a $D(n)$-quadruple for $n \in S$. 
Dujella also considered the existence of $D(a+b \sqrt{-1})$-quadruples in the ring of Gaussian integers. In [6], it was shown that if $b$ is odd or $a \equiv b \equiv 2(\bmod 4)$, then there does not exist a $D(a+b \sqrt{-1})$-quadruple, and if $z=a+b \sqrt{-1}$ is not of that form and $z \notin\{ \pm 2, \pm 1 \pm 2 i, \pm 4 i\}$, then there exist at least two distinct Diophantine quadruples with the property $D(z)$. Franušić also gave some results on Diophantine quadruples in Gaussian integers (see [13]), and in [11,12,14] completely solved the analogous problem in some real quadratic fields.

In addition to results of Dujella and Franušić in Gaussian integers, the case of the ring $\mathbb{Z}[\sqrt{-2}]$ of integers in the quadratic field $\mathbb{Q}(\sqrt{-2})$ is the only case of a complex quadratic field studied until now. It started by the work of Abu Muriefah and Al-Rashed in [1]. Dujella and Soldo extended the results of Abu Muriefah and AlRashed and obtained several new polynomial formulas for Diophantine quadruples with the property $D(a+b \sqrt{-2})$, for integers $a$ and $b$ satisfying certain congruence conditions. Their main result is

Theorem 1 ([10], Theorem 1.1). Let $z \in \mathbb{Z}[\sqrt{-2}]$. If $z$ is of the form $z=a+$ $(2 b+1) \sqrt{-2}$ or $z=4 a+(4 b+2) \sqrt{-2}, a, b \in \mathbb{Z}$, then there does not exist a $D(z)$ quadruple. If $z$ is not of that form, then there exists at least one $D(z)$-quadruple, except maybe if $z$ has one of the following forms:

$$
\begin{aligned}
& z=24 a+2+(12 b+6) \sqrt{-2}, z=24 a+5+(12 b+6) \sqrt{-2}, \\
& z=48 a+44+(24 b+12) \sqrt{-2}
\end{aligned}
$$

or if $z \in\{-1,1 \pm 2 \sqrt{-2}\}$.

In this paper we considered the existence of $D(z)$-quadruple for $z=24 a+2+$ $(12 b+6) \sqrt{-2}, z=24 a+5+(12 b+6) \sqrt{-2}, z=48 a+44+(24 b+12) \sqrt{-2}$, these are cases not covered by the research described in [10]. By [9, Theorem 1], each of these elements can be represented as a difference of two squares of elements in $\mathbb{Z}[\sqrt{-2}]$. Thus, in an analogy to what is known in $\mathbb{Z}$ (see [5]) and certain quadratic fields (see $[6,11,12,14]$ ), it seems reasonable to expect that for such $z$ 's there exists at least one $D(z)$-quadruple, with perhaps finitely many exceptions.

The methods for the construction of Diophantine quadruples usually use elements with small norm. We proved that for such $z$ 's, quadruples cannot contain some elements with very small norm, as in the formulas in Sections 3 and 4 of [10].

Moreover, for finding formulas for Diophantine quadruples, we used the method described in details in [10, Section 2]. Using the fact that numbers 11 and 22 factorizes in $\mathbb{Z}[\sqrt{-2}]$ as $11=(3+\sqrt{-2})(3-\sqrt{-2})$ and $22=(2+3 \sqrt{-2})(2-3 \sqrt{-2})$, we found some formulas for quadruples containing the elements $2 \pm 3 \sqrt{-2}$ with norm 22 and $3 \pm \sqrt{-2}$ with norm 11. By studying them carefully, we obtained our main results, covered by Propositions 3, 4, and 5, presented in Section 3. Namely, we showed the existence of $D(z)$-quadruples for significant proportion of the remaining 
three cases. These new formulas then necessarily involve some congruence conditions modulo 11 on $a$ and $b$, which makes those cases harder to handle.

We also specify very simple result which will be used later on.

Lemma 1. Let $\left\{z_{1}, z_{2}, z_{3}, z_{4}\right\} \subset \mathbb{Z}[\sqrt{-2}]$ be a set with the property $D(z)$ and $w \in \mathbb{Z}[\sqrt{-2}]$. Then

(i) the set $\left\{\bar{z}_{1}, \bar{z}_{2}, \bar{z}_{3}, \bar{z}_{4}\right\}$ has the property $D(\bar{z})$,

(ii) the set $\left\{z_{1} w, z_{2} w, z_{3} w, z_{4} w\right\}$ has the property $D\left(z w^{2}\right)$.

Note that by multiplying elements of a $D(24 a+2+(12 b+6) \sqrt{-2})$-quadruple by $\sqrt{-2}$, by Lemma 1(ii) we obtain (with obvious substitution) a $D(48 a+44+$ $(24 b+12) \sqrt{-2})$-quadruple. Thus, it is sufficient to consider $D(z)$-quadruples for $z=24 a+2+(12 b+6) \sqrt{-2}$ and $z=24 a+5+(12 b+6) \sqrt{-2}$.

\section{ELEMENTS OF SMALL NORM}

Concerning to three unsolved cases from Theorem 1, it can be shown that such quadruples cannot contain some elements with small norm.

Proposition 1. Let $z=24 a+5+(12 b+6) \sqrt{-2}, a, b \in \mathbb{Z}$ and $s \in\{ \pm 1, \pm 3, \pm 4$, $\pm 1 \pm \sqrt{-2}, \pm 2 \pm \sqrt{-2}, \pm 1 \pm 2 \sqrt{-2}\}$. Then there does not exist a $D(z)$-quadruple of the form $\{s, t, u, v\}$ in $\mathbb{Z}[\sqrt{-2}]$.

Proof. Let $z=24 a+5+(12 b+6) \sqrt{-2}, a, b \in \mathbb{Z}$.

(1) Suppose that $s=1$ and $\{1, t, u, v\}$ is $D(z)$-quadruple in $\mathbb{Z}[\sqrt{-2}]$. Then there exists an element $u_{1} \in \mathbb{Z}[\sqrt{-2}]$ such that $t+z=u_{1}^{2}$. We will look this equation modulo 4. Since

$$
\begin{aligned}
u_{1}^{2} & \equiv 0,1,2,3+2 \sqrt{-2} \quad(\bmod 4), \\
z & \equiv 1+2 \sqrt{-2} \quad(\bmod 4),
\end{aligned}
$$

from $t=u_{1}^{2}-z$, we obtain $t \equiv t^{\prime}(\bmod 4)$, where

$$
t^{\prime} \in S=\{2,2 \sqrt{-2}, 1+2 \sqrt{-2}, 3+2 \sqrt{-2}\} .
$$

Similarly, $u \equiv u^{\prime}(\bmod 4), v \equiv v^{\prime}(\bmod 4)$, where $u^{\prime}, v^{\prime} \in S$.

On the other hand, there exist $u_{2}, u_{3}, u_{4} \in \mathbb{Z}[\sqrt{-2}]$, such that $t u+z=u_{2}^{2}, t v+$ $z=u_{3}^{2}, u v+z=u_{4}^{2}$. In a same way, we obtain $t u \equiv(t u)^{\prime}(\bmod 4), t v \equiv(t v)^{\prime}$ $(\bmod 4), u v \equiv(u v)^{\prime}(\bmod 4)$, where $(t u)^{\prime},(t s)^{\prime},(u s)^{\prime} \in S$.

This means that there exist three (equal or distinct) elements of the set $S$, such that the product of any two of them is some element of $S$. It is easy to check that this is not possible.

From Lema 1(ii) for $w=-1$, it follows that there does not exist a $D(z)$-quadruple of the form $\{-1, t, u, v\}$. 
(2) If we suppose that $s=4$ and $\{4, t, u, v\}$ is $D(z)$-quadruple in $\mathbb{Z}[\sqrt{-2}]$, then there exists an element $w_{1} \in \mathbb{Z}[\sqrt{-2}]$ such that $4 t+z=w_{1}^{2}$. Since, $w_{1}^{2}-z \equiv t_{1}$ $(\bmod 4)$, where $t_{1} \in S$, from $4 t=w_{1}^{2}-z$ we obtain a contradiction. The nonexistence of a $D(z)$-quadruple of the form $\{-4, t, u, v\}$ immediately follows from Lemma 1(ii).

(3) Let $s=1 \pm \sqrt{-2}$ and $\{s, t, u, v\}$ be a $D(z)$-quadruple in $\mathbb{Z}[\sqrt{-2}]$. Then there exists an element $w_{2} \in \mathbb{Z}[\sqrt{-2}]$ such that $s t+z=w_{2}^{2}$. We have

$$
\begin{aligned}
w^{2} & \equiv 0,1 \quad(\bmod s), \\
z & \equiv 2 \quad(\bmod s) .
\end{aligned}
$$

Then from $s t=w_{2}^{2}-z$ we get a contradiction.

Since $1+\sqrt{-2}$ or $1-\sqrt{-2}$ is a divisor of any element of the set

$$
T=\{ \pm 3,-1 \pm \sqrt{-2}, \pm 2 \pm \sqrt{-2}, \pm 1 \pm 2 \sqrt{-2}\},
$$

we conclude that there does not exist $D(z)$-quadruple of the form $\{s, t, u, v\}, s \in$ $T$.

Proposition 2. Let $z=24 a+2+(12 b+6) \sqrt{-2}, a, b \in \mathbb{Z}$ and $s \in\{ \pm 1, \pm 3, \pm 4$, $\pm 1 \pm \sqrt{-2}, \pm 2 \pm \sqrt{-2}, \pm 1 \pm 2 \sqrt{-2}\}$. Then there does not exist a $D(z)$-quadruple of the form $\{s, t, u, v\}$ in $\mathbb{Z}[\sqrt{-2}]$.

Proof. Let $z=24 a+2+(12 b+6) \sqrt{-2}, a, b \in \mathbb{Z}$.

(1) Suppose that $s=1$ and $\{1, t, u, v\}$ is $D(z)$-quadruple in $\mathbb{Z}[\sqrt{-2}]$. Then there exists an element $u_{1} \in \mathbb{Z}[\sqrt{-2}]$ such that $t+z=u_{1}^{2}$. We will look this equation modulo 8 . We have

$$
u_{1}^{2} \equiv 0,1,4,6,1+4 \sqrt{-2}, 2+4 \sqrt{-2}, 7+2 \sqrt{-2}, 7+6 \sqrt{-2} \quad(\bmod 8) .
$$

From $12 b+6 \equiv 2,6(\bmod 8)$, we conclude:

(i) If $z \equiv 2+2 \sqrt{-2}(\bmod 8)$, then from $t=u_{1}^{2}-z$ we get $t \equiv t^{\prime}(\bmod 8)$, where

$$
\begin{gathered}
t^{\prime} \in S_{1} \\
=\{5,2 \sqrt{-2}, 7+2 \sqrt{-2}, 5+4 \sqrt{-2}, 2+6 \sqrt{-2}, 4+6 \sqrt{-2}, 6+6 \sqrt{-2}, 7+6 \sqrt{-2}\} .
\end{gathered}
$$

Similarly, $u \equiv u^{\prime}(\bmod 8), v \equiv v^{\prime}(\bmod 8), t u \equiv(t u)^{\prime}(\bmod 8), t v \equiv(t v)^{\prime}(\bmod 8)$, $u v \equiv(u v)^{\prime}(\bmod 8)$, where $u^{\prime}, v^{\prime},(t u)^{\prime},(t v)^{\prime},(u v)^{\prime} \in S_{1}$.

(ii) If $z \equiv 2+6 \sqrt{-2}(\bmod 8)$, in the same way we get $t^{\prime}, u^{\prime}, v^{\prime},(t u)^{\prime},(t v)^{\prime},(u v)^{\prime} \in$ $S_{2}$, where

$S_{2}=\{5,6 \sqrt{-2}, 2+2 \sqrt{-2}, 4+2 \sqrt{-2}, 6+2 \sqrt{-2}, 7+2 \sqrt{-2}, 5+4 \sqrt{-2}, 7+6 \sqrt{-2}\}$.

Now, in both cases, it is easy to check that there does not exist three (equal or distinct) elements of the sets $S_{1}$ and $S_{2}$, such that the product of any two of them is some element of those sets. Using the Lema 1(ii), we conclude that there does not exist a $D(z)$-quadruple of the form $\{-1, t, u, v\}$. 
(2) If we suppose that $s=4$ and $\{4, t, u, v\}$ is $D(z)$-quadruple in $\mathbb{Z}[\sqrt{-2}]$, then there exists an element $w_{1} \in \mathbb{Z}[\sqrt{-2}]$ such that $4 t+z=w_{1}^{2}$. Since,

$$
\begin{aligned}
w_{1}^{2} & \equiv 0,1,2,3+2 \sqrt{-2} \quad(\bmod 4), \\
z & \equiv 2+2 \sqrt{-2} \quad(\bmod 4),
\end{aligned}
$$

we conclude that $w_{1}^{2}-z \equiv 1,2 \sqrt{-2}, 2+2 \sqrt{-2}, 3+2 \sqrt{-2}(\bmod 4)$. Now, from $4 t=w_{1}^{2}-z$, we get a contradiction. Now, Lema 1(ii) implies that there does not exist a $D(z)$-quadruple of the form $\{-4, t, u, v\}$.

(3) Let $s=1 \pm \sqrt{-2}$ and $\{s, t, u, v\}$ be a $D(z)$-quadruple in $\mathbb{Z}[\sqrt{-2}]$. Since $z \equiv 2$ $(\bmod s)$, the proof is the same as in case (3) in Proposition 1.

On the other hand, by following the approach from [7], we were able to prove that there exist infinitely many $D(z)$-quadruples for $z$ of studied forms, containing elements \pm 2 and $\pm 2 \sqrt{-2}$.

We consider the existence of $D(z)$-quadruples of the form $\{u, v, u+v+2 r, 4 u+$ $v+4 r\}, u, v, r \in \mathbb{Z}[\sqrt{-2}]$. The method is based on factorization $z=s t, s, t \in$ $\mathbb{Z}[\sqrt{-2}]$. For arbitrary $u$, let $v$ be an element such that $u v+z=r^{2}$. The set $\{u, v, u+v+2 r\}$ has the property $D(z)$. Indeed,

$$
\begin{aligned}
& u(u+v+2 r)+z=(u+r)^{2}, \\
& v(u+v+2 r)+z=(v+r)^{2} .
\end{aligned}
$$

If we apply this construction to the Diophantine pair $\{u, u+v+2 r\}$ we obtain the set $\{u, u+v+2 r, 4 u+v+4 r\}$ also with the property $D(z)$. Therefore, the set

$$
\{u, v, u+v+2 r, 4 u+v+4 r\}
$$

has the property $D(z)$, if and only if

$$
v(4 u+v+4 r)+z=y^{2}, \quad y \in \mathbb{Z}[\sqrt{-2}] .
$$

That is equivalent to

$$
(v+2 r-y)(v+2 r+y)=3 s t .
$$

If we set

$$
\begin{aligned}
& v+2 r-y=s, \\
& v+2 r+y=3 t,
\end{aligned}
$$

we obtain

$$
2 v+4 r=s+3 t .
$$

In the first case we set $u=2$. Using $2 v+z=r^{2}$, from (2.2) it follows that

$$
(r+2)^{2}=(s+3)(t+1)+1 .
$$

Now, we can choose $r+2=(t+1) m \pm 1$ or $r+2=(s+3) m \pm 1$, for $m \in \mathbb{Z}[\sqrt{-2}]$.

Let $r+2=(s+3) m-1$. Then

$$
t+1=(s+3) m^{2}-2 m,
$$




$$
z=s t=s(s+3) m^{2}-(2 m+1) s .
$$

If we choose $m=1-\sqrt{-2}$ and $s=6 p+4+(6 q+3) \sqrt{-2}, p, q \in \mathbb{Z}$, we obtain the set

$$
\begin{aligned}
& \{2,-18 p+12 q-15+(-6 p-18 q-13) \sqrt{-2} \\
& -6 p+36 q+7+(-18 p-6 q-21) \sqrt{-2} \\
& 6 p+60 q+33+(-30 p+6 q-29) \sqrt{-2}\}
\end{aligned}
$$

with the property

$$
\begin{aligned}
& D\left(60 p-36 p^{2}+312 q+288 p q+72 q^{2}+98\right. \\
& \left.\quad+\left(-156 p-72 p^{2}+60 q-72 p q+144 q^{2}-54\right) \sqrt{-2}\right) .
\end{aligned}
$$

Let $r+2=(t+1) m+1$. For $m=2$ and $t=24 p+5+(12 q+6) \sqrt{-2}, p, q \in \mathbb{Z}$, we get the set

$$
\begin{aligned}
& \{2,-12 p-2+(-6 q-3) \sqrt{-2}, \\
& 84 p+22+(42 q+21) \sqrt{-2}, \\
& 180 p+50+(90 q+45) \sqrt{-2}\},
\end{aligned}
$$

with the property

$$
\begin{aligned}
& D\left(1080 p+2304 p^{2}-1152 q-1152 q^{2}-163\right. \\
& +(1152 p+540 q+2304 p q+270) \sqrt{-2}) .
\end{aligned}
$$

Now, let $u=2 \sqrt{-2}$. From (2.1) and $2 \sqrt{-2} v+z=r^{2}$, we obtain

$$
(r+2 \sqrt{-2})^{2}=(s+3 \sqrt{-2})(t+\sqrt{-2})-2 .
$$

If we set $r+2 \sqrt{-2}=(s+3 \sqrt{-2}) m+\sqrt{-2}$, for $m=2-\sqrt{-2}$ and $s=12 p+6+$ $(12 q+1) \sqrt{-2}, p, q \in \mathbb{Z}$, we get the set

$$
\begin{aligned}
& \{2 \sqrt{-2},-6 p+96 q+35+(-48 p-6 q-21) \sqrt{-2}, \\
& 42 p+144 q+75+(-72 p+42 q-17) \sqrt{-2}, \\
& 90 p+192 q+115+(-96 p+90 q-9) \sqrt{-2}\},
\end{aligned}
$$

with the property

$$
\begin{aligned}
& D\left(816 p+288 p^{2}+840 q+2304 p q-576 q^{2}+314\right. \\
& \left.\quad+\left(-420 p-576 p^{2}+816 q+576 p q+1152 q^{2}-30\right) \sqrt{-2}\right) .
\end{aligned}
$$

Similarly, if $r+2 \sqrt{-2}=(s+3 \sqrt{-2}) m-\sqrt{-2}$, for $m=1-\sqrt{-2}$ and $s=6 p+$ $3+(6 q+1) \sqrt{-2}, p, q \in \mathbb{Z}$, we obtain the set

$$
\begin{aligned}
& \{2 \sqrt{-2},-18 p+12 q-7+(-6 p-18 q-15) \sqrt{-2}, \\
& -6 p+36 q+15+(-18 p-6 q-17) \sqrt{-2}, \\
& 6 p+60 q+37+(-30 p+6 q-15) \sqrt{-2}\},
\end{aligned}
$$


with the property

$$
\begin{aligned}
& D\left(60 p-36 p^{2}+240 q+288 p q+72 q^{2}+53\right. \\
& \left.\quad+\left(-120 p-72 p^{2}+60 q-72 p q+144 q^{2}-30\right) \sqrt{-2}\right) .
\end{aligned}
$$

By Lemma 1(ii) we obtain $D(z)$-quadruples with elements $-2,-2 \sqrt{-2}$.

Remark 1. While, for example, the property

$$
\begin{aligned}
& D\left(1080 p+2304 p^{2}-1152 q-1152 q^{2}-163\right. \\
& +(1152 p+540 q+2304 p q+270) \sqrt{-2})
\end{aligned}
$$

of the set (2.4) can be expressed as $D(24 a+5+(12 b+6) \sqrt{-2})$, where

$$
\begin{aligned}
& a=45 p+96 p^{2}-48 q-48 q^{2}-7, \\
& b=96 p+45 q+192 p q+22,
\end{aligned}
$$

for the most of integer pairs $(a, b)$ there does not exist integers $p$ and $q$ such that $a$ and $b$ can be represented by (2.7). Similar situation appears while considering properties of all the other sets. This means that obtained results do not cover any of the remaining unsolved cases of Theorem 1 (regardless of the fact that for arbitrary $p$ an $q$ such sets generate infinitely many quadruples with the certain property).

\section{3. $D(z)$-QUADRUPLES WITH AN ELEMENT OF NORM 11}

In this section, according to three unsolved cases

$$
\begin{aligned}
& z=24 a+2+(12 b+6) \sqrt{-2}, z=24 a+5+(12 b+6) \sqrt{-2}, \\
& z=48 a+44+(24 b+12) \sqrt{-2},
\end{aligned}
$$

of Theorem 1, we consider the existence of a $D(z)$-quadruple of the form $\{u, v, u+$ $v+2 r, u+4 v+4 v\}, u, v, r \in \mathbb{Z}[\sqrt{-2}]$. We obtain some new results by considering $a$ and $b$ modulo 11, using the method in detail described in [10, Section 2]. Anyway, here we will specify that method again, for the convenience of the reader.

Let $\{u, v\}$ be an arbitrary pair with the property $D(z)$, for $z \in \mathbb{Z}[\sqrt{-2}]$. It means that

$$
u v+z=r^{2}
$$

for $r \in \mathbb{Z}[\sqrt{-2}]$. Similarly as in Section 2, the only condition such that the set

$$
\{u, v, u+v+2 r, u+4 v+4 r\}
$$

has the property $D(z)$ is equal to

$$
u(u+4 v+4 r)+z=y^{2},
$$

i.e.

$$
3 z=(u+2 r-y)(u+2 r+y) .
$$

This means that there is an element $e \in \mathbb{Z}[\sqrt{-2}]$ such that

$$
u+2 r-y=e,
$$




$$
u+2 r+y=\frac{3 z}{e}
$$

which gives

$$
2 u+4 r=\frac{3 z \bar{e}}{N(e)}+e,
$$

where $\bar{e}$ is the conjugate of an element $e$ and $N(e)=e \cdot \bar{e}$ is the norm of $e$. Suppose that $z$ and $e$ are given. Then, if we look at equation (3.2) modulo 4, we get a condition for the form of $u$. We choose a $u$ of small norm satisfying this condition. Now, it is easy to find the form of $r$. It remains to satisfy the condition that $v=\left(r^{2}-z\right) / u \in$ $\mathbb{Z}[\sqrt{-2}]$, which is equivalent to the divisibility condition

$$
N(u) \mid\left(r^{2}-z\right) \cdot \bar{u}
$$

Proposition 3. If $z$ is of the form $z=24 a+5+(12 b+6) \sqrt{-2}$, then there exists at least one Diophantine quadruple with the property $D(z)$, for any $a, b \in \mathbb{Z}$, except maybe for $a \equiv a^{\prime}(\bmod 11), b \equiv b^{\prime}(\bmod 11)$, where

$$
\begin{aligned}
\left(a^{\prime}, b^{\prime}\right) & \in\{(0,3),(0,4),(0,6),(0,7),(1,1),(1,5),(1,9),(2,1),(2,2),(2,8), \\
& (2,9),(3,5),(4,3),(4,5),(4,7),(5,0),(5,2),(5,8),(5,10),(6,0), \\
& (6,5),(6,10),(7,2),(7,5),(7,8),(8,4),(8,5),(8,6),(9,1),(9,3), \\
& (9,7),(9,9),(10,0),(10,4),(10,6),(10,10)\} .
\end{aligned}
$$

Proof. Let $z=24 a+5+(12 b+6) \sqrt{-2}, a, b \in \mathbb{Z}$, and $e=3$. We choose $u=$ $2+3 \sqrt{-2}$ and $N(u)=22$. Now, from (3.2), it follows that

$$
r=6 a+1+3 b \sqrt{-2} .
$$

From the condition $u v+z=r^{2}$, it follows that

$$
\begin{aligned}
v= & \frac{1}{11}\left(36 a^{2}-12 a+108 a b-18 b^{2}-18 b-22\right) \\
& +\frac{1}{11}\left(-54 a^{2}+18 a+36 a b+27 b^{2}-6 b\right) \sqrt{-2} .
\end{aligned}
$$


We get the set

$$
\begin{aligned}
& \left\{2+3 \sqrt{-2}, \frac{1}{11}\left(36 a^{2}-12 a+108 a b-18 b^{2}-18 b-22\right)\right. \\
& +\frac{1}{11}\left(-54 a^{2}+18 a+36 a b+27 b^{2}-6 b\right) \sqrt{-2}, \\
& \frac{1}{11}\left(36 a^{2}+120 a+108 a b-18 b^{2}-18 b+22\right) \\
& +\frac{1}{11}\left(-54 a^{2}+18 a+36 a b+27 b^{2}+60 b+33\right) \sqrt{-2}, \\
& \frac{1}{11}\left(144 a^{2}+216 a+432 a b-72 b^{2}-72 b-22\right) \\
& \left.+\frac{1}{11}\left(-216 a^{2}+72 a+144 a b+108 b^{2}+108 b+33\right) \sqrt{-2}\right\},
\end{aligned}
$$

with the property $D(z)$ in $\mathbb{Q}(\sqrt{-2})$. It remains to check for which pairs $(a, b)$ modulo 11, set (3.3) has the elements in the ring $\mathbb{Z}[\sqrt{-2}]$. Equivalently, we have to find all pairs $(a, b)$ which satisfy the condition

$$
N(u) \mid\left(r^{2}-z\right) \cdot \bar{u},
$$

i.e.

$$
\begin{aligned}
72 a^{2}-24 a+216 a b-36 b^{2}-36 b-44 & \equiv 0 & & (\bmod 11), \\
-108 a^{2}+36 a+72 a b+54 b^{2}-12 b & \equiv 0 & & (\bmod 11) .
\end{aligned}
$$

It is easy to check that the above condition is satisfied for all $(a, b)=\left(11 k+a^{\prime}, 11 l+\right.$ $\left.b^{\prime}\right), k, l \in \mathbb{Z}$, where

$$
\begin{aligned}
\left(a^{\prime}, b^{\prime}\right) & \in\{(0,0),(0,10),(1,2),(1,3),(2,5),(2,6),(3,8),(3,9),(4,0),(4,1), \\
& (5,3),(5,4),(6,6),(6,7),(7,9),(7,10),(8,1),(8,2),(9,4),(9,5) \\
& (10,7),(10,8)\}
\end{aligned}
$$

Let us determine the pairs $(a, b)$ for which set (3.3) has at least two equal elements or some elements equal to zero. If $(a, b)=(11 k, 11 l)$, the above cases appear if $(k, l)=(0,0)$. But for that pair the set $\{1-3 \sqrt{-2},-3+\sqrt{-2}, 6+2 \sqrt{-2}, 5+9 \sqrt{-2}\}$ has the property $D(5+6 \sqrt{-2})$. If $(a, b)=(11 k, 11 l+10)$, the above cases appear if $(k, l)=(0,-1)$. But in this case, the set $\{1+3 \sqrt{-2},-3-\sqrt{-2}, 6-2 \sqrt{-2}, 5-$ $9 \sqrt{-2}\}$ has the property $D(5-6 \sqrt{-2})$.

Similarly, if $u=2-3 \sqrt{-2}$, we obtain another set with the property $D(z)$ in $\mathbb{Q}(\sqrt{-2})$. The set has the elements in the ring $\mathbb{Z}[\sqrt{-2}]$ for new pairs $(a, b)=$ $\left(11 k+a^{\prime}, 11 l+b^{\prime}\right), k, l \in \mathbb{Z}$, where

$$
\begin{gathered}
\left(a^{\prime}, b^{\prime}\right) \in\{(1,7),(1,8),(2,4),(3,1),(3,2),(4,9),(4,10),(5,6),(5,7),(6,3), \\
(6,4),(7,0),(7,1),(8,8),(8,9),(9,6),(10,2),(10,3)\},
\end{gathered}
$$

and contains four distinct elements. 
Let $e=1+\sqrt{-2}$ and $u=3+\sqrt{-2}$ with $N(u)=11$. In the similar way, we also get a set with the property $D(z)$ in $\mathbb{Q}(\sqrt{-2})$. If $(a, b)=\left(11 k+a^{\prime}, 11 l+b^{\prime}\right), k, l \in \mathbb{Z}$, and

$$
\left(a^{\prime}, b^{\prime}\right) \in\{(0,9),(1,6),(2,3),(3,0),(4,8),(5,5),(6,2),(8,7),(10,1)\},
$$

then the set contains four distinct elements in the ring $\mathbb{Z}[\sqrt{-2}]$. If we put $u=3-$ $\sqrt{-2}$, a new set has all distinct elements in the ring $\mathbb{Z}[\sqrt{-2}]$ for new pairs $(a, b)=$ $\left(11 k+a^{\prime}, 11 l+b^{\prime}\right), k, l \in \mathbb{Z}$ and

$$
\begin{gathered}
\left(a^{\prime}, b^{\prime}\right) \in\{(0,5),(0,8),(1,0),(2,0),(3,3),(3,6),(4,6),(5,1),(5,9),(6,1), \\
(7,4),(7,7),(8,10),(9,2),(9,10),(10,5)\} .
\end{gathered}
$$

Now, we take $e=1-\sqrt{-2}$ and $u=3+\sqrt{-2}$. We obtain another set with the property $D(z)$ in $\mathbb{Q}(\sqrt{-2})$. It is easy to check that for all $(a, b)=\left(11 k+a^{\prime}, 11 l+\right.$ $\left.b^{\prime}\right), k, l \in \mathbb{Z}$, where

$$
\begin{gathered}
\left(a^{\prime}, b^{\prime}\right) \in\{(0,2),(1,10),(2,7),(2,10),(3,4),(3,7),(4,4),(6,9),(7,3), \\
(7,6),(8,0),(8,3),(9,0),(9,8)\},
\end{gathered}
$$

the set contains four distinct elements in the ring $\mathbb{Z}[\sqrt{-2}]$. Finally, if we choose $u=3-\sqrt{-2}$, we get the new set and new pairs $(a, b)=\left(11 k+a^{\prime}, 11 l+b^{\prime}\right)$,

$$
\left(a^{\prime}, b^{\prime}\right) \in\{(0,1),(1,4),(3,10),(4,2),(6,8),(10,9)\},
$$

with four distinct elements in the ring $\mathbb{Z}[\sqrt{-2}]$.

Proposition 4. If $z$ is of the form $z=24 a+2+(12 b+6) \sqrt{-2}$, then there exists at least one Diophantine quadruple with the property $D(z)$, for any $a, b \in \mathbb{Z}$, except maybe for $a \equiv a^{\prime}(\bmod 11), b \equiv b^{\prime}(\bmod 11)$, where

$$
\begin{aligned}
\left(a^{\prime}, b^{\prime}\right) & \in\{(0,3),(0,5),(0,7),(1,0),(1,2),(1,8),(1,10),(2,0),(2,5),(2,10), \\
& (3,2),(3,5),(3,8),(4,4),(4,5),(4,6),(5,1),(5,3),(5,7),(5,9), \\
& (6,0),(6,4),(6,6),(6,10),(7,3),(7,4),(7,6),(7,7),(8,1),(8,5), \\
& (8,9),(9,1),(9,2),(9,8),(9,9),(10,5)\} .
\end{aligned}
$$


Proof. Let $z=24 a+2+(12 b+6) \sqrt{-2}, a, b \in \mathbb{Z}$, and $e=2+\sqrt{-2}$. We choose $u=3-\sqrt{-2}$ and $N(u)=11$. We get the set

$$
\begin{aligned}
& \left\{3-\sqrt{-2}, \frac{1}{11}\left(126 a^{2}+180 a b-63 b^{2}-66 b-23\right)\right. \\
& +\frac{1}{11}\left(-90 a^{2}+66 a+126 a b+45 b^{2}-15\right) \sqrt{-2}, \\
& \frac{1}{11}\left(126 a^{2}+132 a+180 a b-63 b^{2}-32\right) \\
& +\frac{1}{11}\left(-90 a^{2}+126 a b+45 b^{2}-66 b+18\right) \sqrt{-2}, \\
& \frac{1}{11}\left(504 a^{2}+264 a+720 a b-252 b^{2}-132 b-15\right) \\
& \left.+\frac{1}{11}\left(-360 a^{2}+132 a+504 a b+180 b^{2}+132 b+17\right) \sqrt{-2}\right\},
\end{aligned}
$$

with the property $D(z)$ in $\mathbb{Q}(\sqrt{-2})$. It is easy to check that the set (3.4) has all distinct elements in the ring $\mathbb{Z}[\sqrt{-2}]$ for all $(a, b)=\left(11 k+a^{\prime}, 11 l+b^{\prime}\right), k, l \in \mathbb{Z}$, where

$$
\begin{aligned}
\left(a^{\prime}, b^{\prime}\right) & \in\{(0,2),(0,9),(1,1),(1,5),(2,4),(2,8),(3,0),(3,7),(4,3),(4,10), \\
& (5,2),(5,6),(6,5),(6,9),(7,1),(7,8),(8,0),(8,4),(9,3),(9,7), \\
& (10,6),(10,10)\} .
\end{aligned}
$$

Similarly, if $u=3+\sqrt{-2}$, we obtain another set with the property $D(z)$ in $\mathbb{Q}(\sqrt{-2})$. That set has the elements in the ring $\mathbb{Z}[\sqrt{-2}]$ for new pairs $(a, b)=\left(11 k+a^{\prime}, 11 l+\right.$ $\left.b^{\prime}\right), k, l \in \mathbb{Z}$, where

$$
\begin{array}{r}
\left(a^{\prime}, b^{\prime}\right) \in\{(0,4),(1,6),(2,3),(2,9),(3,6),(4,8),(5,0),(5,5),(6,2), \\
(6,8),(7,5),(7,10),(8,2),(8,7),(9,4),(9,10),(10,1),(10,7)\},
\end{array}
$$

and contains four distinct elements.

Let $e=2-\sqrt{-2}$ and $u=3+\sqrt{-2}$. In a same way, we also get a set with the property $D(z)$ in $\mathbb{Q}(\sqrt{-2})$. If $(a, b)=\left(11 k+a^{\prime}, 11 l+b^{\prime}\right), k, l \in \mathbb{Z}$, and

$$
\begin{array}{r}
\left(a^{\prime}, b^{\prime}\right) \in\{(0,1),(0,8),(1,9),(2,2),(2,6),(3,3),(3,10),(4,0),(4,7), \\
(5,4),(5,8),(6,1),(7,2),(7,9),(8,6),(8,10),(10,0),(10,4)\},
\end{array}
$$

then the set contains four distinct elements in the ring $\mathbb{Z}[\sqrt{-2}]$. If we put $u=3-$ $\sqrt{-2}$, a new set has all distinct elements in the ring $\mathbb{Z}[\sqrt{-2}]$ for new pairs $(a, b)=$ $\left(11 k+a^{\prime}, 11 l+b^{\prime}\right), k, l \in \mathbb{Z}$ and

$$
\begin{array}{r}
\left(a^{\prime}, b^{\prime}\right) \in\{(0,6),(1,4),(2,1),(2,7),(3,4),(4,2),(5,10), \\
(7,0),(8,3),(8,8),(9,0),(9,6),(10,3),(10,9)\} .
\end{array}
$$


Now, we take $e=\sqrt{-2}$ and $u=3-\sqrt{-2}$. We obtain another set with the property $D(z)$ in $\mathbb{Q}(\sqrt{-2})$. It is easy to check that for all $(a, b)=\left(11 k+a^{\prime}, 11 l+b^{\prime}\right), k, l \in$ $\mathbb{Z}$, where

$$
\left(a^{\prime}, b^{\prime}\right) \in\{(0,0),(1,3),(3,9),(4,1),(6,7),(9,5),(10,8)\},
$$

the set contains elements of the ring $\mathbb{Z}[\sqrt{-2}]$. If $(a, b)=(11 k, 11 l)$, then there appear two equal elements for $(k, l)=(0,0)$. But for that pair the set $\{1-3 \sqrt{-2}, 2 \sqrt{-2}$, $9+\sqrt{-2}, 17+9 \sqrt{-2}\}$ has the property $D(2+6 \sqrt{-2})$. If we choose $u=3+\sqrt{-2}$, we get the new set and new pairs $(a, b)=\left(11 k+a^{\prime}, 11 l+b^{\prime}\right)$,

$$
\left(a^{\prime}, b^{\prime}\right) \in\{(0,10),(1,7),(3,1),(4,9),(6,3),(10,2)\},
$$

with all elements in the ring $\mathbb{Z}[\sqrt{-2}]$. Moreover, if $(a, b)=(11 k, 11 l+10)$, then the set contains two equal elements for $(k, l)=(0,-1)$. But the set $\{1+3 \sqrt{-2},-2 \sqrt{-2}$, $9-\sqrt{-2}, 17-9 \sqrt{-2}\}$ has the property $D(2-6 \sqrt{-2})$.

As we mentioned, Proposition 4 immediately implies

Proposition 5. If $z$ is of the form $z=48 a+44+(24 b+12) \sqrt{-2}$, then there exists at least one Diophantine quadruple with the property $D(z)$, for any $a, b \in \mathbb{Z}$, except maybe for $a \equiv a^{\prime}(\bmod 11), b \equiv b^{\prime}(\bmod 11)$, where

$$
\begin{aligned}
\left(a^{\prime}, b^{\prime}\right) & \in\{(0,5),(1,1),(1,2),(1,8),(1,9),(2,1),(2,5),(2,9),(3,3),(3,4), \\
& (3,6),(3,7),(4,0),(4,4),(4,6),(4,10),(5,1),(5,3),(5,7),(5,9), \\
& (6,4),(6,5),(6,6),(7,2),(7,5),(7,8),(8,0),(8,5),(8,10),(9,0), \\
& (9,2),(9,8),(9,10),(10,3),(10,5),(10,7)\} .
\end{aligned}
$$

Remark 2. Concerning the possible exceptions from Propositions 3, 4, and 5, for certain numbers $z$ (e.g. $z=5+42 \sqrt{-2}, 5+78 \sqrt{-2}, 29+114 \sqrt{-2}, 197+$ $78 \sqrt{-2}$ of the exceptional form in Proposition 3 ) we were not able to find any $D(z)$ quadruple.

On the other hand, for all exceptional pairs $\left(a^{\prime}, b^{\prime}\right)$ from Proposition 3, we were able to find at least one $z$ of the form $z=24 a+5+(12 b+6) \sqrt{-2}), a \equiv a^{\prime}(\bmod 11)$, $b \equiv b^{\prime}(\bmod 11)$ for which there exist a $D(z)$-quadruple (in some cases we can find infinitely many such $z$ 's by using formulas from Remark 1 ). Some of the observed quadruples contain an element of norm 19. A systematic study of such quadruples would involve additional congruence conditions modulo 19 on $a$ and $b$, so the results become too complicated to state explicitly and do not lead to the complete solution of the problem. Similar results and problems appear when considering exceptional cases from Propositions 4, and 5. 


\section{ACKNOWLEDGEMENT}

The author would like to thank professor Andrej Dujella for both proposing the problem and for his valuable advices on how to attack it. He is also very grateful to the referee for carefully reading of the paper and for all insightful comments and valuable suggestions which improved this paper.

\section{REFERENCES}

[1] F. S. Abu Muriefah and A. Al-Rashed, "Some Diophantine quadruples in the ring $\mathbb{Z}[\sqrt{-2}]$, , Math. Commun., vol. 9, no. 1, pp. 1-8, 2004.

[2] A. Baker and H. Davenport, "The equations $3 x^{2}-2=y^{2}$ and $8 x^{2}-7=z^{2}$," Q. J. Math., Oxf. II. Ser., vol. 20, pp. 129-137, 1969.

[3] E. Brown, "Sets in which $x y+k$ is always a square," Math. Comput., vol. 45, pp. 613-620, 1985.

[4] Diophantus of Alexandria, Arithmetics and the Book of Polygonal Numbers, I. G. Bashmakova, Ed. Moscow: Nauka, 1974.

[5] A. Dujella, "Generalization of a problem of Diophantus," Acta Arith., vol. 65, no. 1, pp. 15-27, 1993.

[6] A. Dujella, "The problem of Diophantus and Davenport for Gaussian integers," Glas. Mat., III. Ser. vol. 32, no. 1, pp. 1-10, 1997.

[7] A. Dujella, "A problem of Diophantus and Dickson's conjecture," in Number theory. Diophantine, computational and algebraic aspects, ser. Proceedings of the international conference, Eger, Hungary, July 29-August 2, 1996. Berlin: de Gruyter, 1998, pp. 147-156.

[8] A. Dujella, "There are only finitely many Diophantine quintuples," J. Reine Angew. Math., vol. 566, pp. 183-214, 2004.

[9] A. Dujella and Z. Franušić, "On differences of two squares in some quadratic fields," Rocky Mt. J. Math., vol. 37, no. 2, pp. 429-453, 2007.

[10] A. Dujella and I. Soldo, "Diophantine quadruples in $\mathbb{Z}[\sqrt{-2}]$," An. Ştiinţ. Univ. "Ovidius" Constanţa, Ser. Mat., vol. 18, no. 1, pp. 81-98, 2010.

[11] Z. Franušić, "Diophantine quadruples in the ring $\mathbb{Z}[\sqrt{s}]$," Math. Commun., vol. 9, no. 2, pp. 141$148,2004$.

[12] Z. Franušić, "Diophantine quadruples in $\mathbb{Z}[\sqrt{4 k+3}]$," Ramanujan J., vol. 17, no. 1, pp. 77-88, 2008.

[13] Z. Franušić, "On the extensibility of Diophantine triples $\{k-1, k+1,4 k\}$ for Gaussian integers," Glas. Mat., III. Ser., vol. 43, no. 2, pp. 265-291, 2008.

[14] Z. Franušić, “A Diophantine problem in $\mathbb{Z}[(1+\sqrt{d}) / 2]$," Stud. Sci. Math. Hung., vol. 46, no. 1, pp. 103-112, 2009.

[15] T. L. Heath, Diophantus of Alexandria. A Study in the History of Greek Algebra. Powell's Bookstore, Chicago: Martino Publishing, Mansfield Center, 2003.

\section{Author's address}

\section{Ivan Soldo}

University of Osijek, Department of Mathematics, Trg Ljudevita Gaja 6, 31000 Osijek, Croatia

E-mail address: isoldo@mathos.hr 